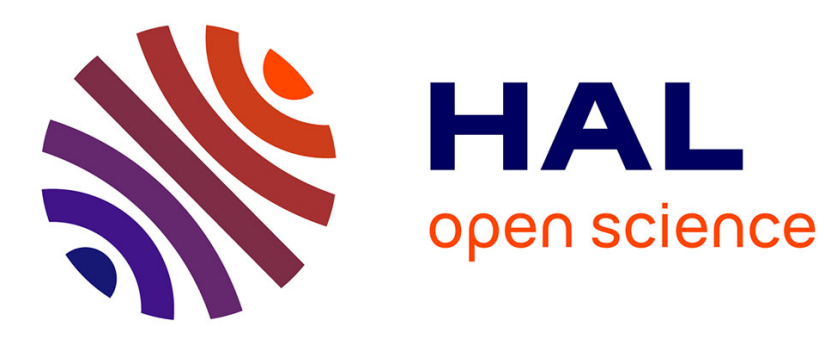

\title{
A mesoscopic rheological model of moderately concentrated colloids
}

Miroslav Grmela, Amine Ammar, Francisco Chinesta, Guillaume Maitrejean

\section{To cite this version:}

Miroslav Grmela, Amine Ammar, Francisco Chinesta, Guillaume Maitrejean. A mesoscopic rheological model of moderately concentrated colloids. Journal of Non-Newtonian Fluid Mechanics, 2014, 212, pp.1-12. 10.1016/j.jnnfm.2014.07.005 . hal-02465887

\section{HAL Id: hal-02465887 \\ https://hal.science/hal-02465887}

Submitted on 4 Feb 2020

HAL is a multi-disciplinary open access archive for the deposit and dissemination of scientific research documents, whether they are published or not. The documents may come from teaching and research institutions in France or abroad, or from public or private research centers.
L'archive ouverte pluridisciplinaire HAL, est destinée au dépôt et à la diffusion de documents scientifiques de niveau recherche, publiés ou non, émanant des établissements d'enseignement et de recherche français ou étrangers, des laboratoires publics ou privés. 


\title{
A mesoscopic rheological model of moderately concentrated colloids
}

\author{
Miroslav Grmela ${ }^{\mathrm{a}, *}$, Amine Ammar $^{\mathrm{b}}$, Francisco Chinesta ${ }^{\mathrm{c}}$, Guillaume Maîtrejean ${ }^{\mathrm{d}}$ \\ ${ }^{a}$ Ecole Polytechnique de Montreal, C.P.6079 suc. Centre-ville, Montreal, H3C 3A7 Quebec, Canada \\ ${ }^{\mathrm{b}}$ Arts et Métiers ParisTech, 2 Boulevard du Ronceray, BP 93525, 49035 Angers Cedex 01, France \\ ${ }^{\mathrm{c}}$ EADS Corporate International Chair GeM, Ecole Centrale de Nantes, BP 92101, 44321 Nantes Cedex 3, France \\ ${ }^{\mathrm{d}}$ Laboratoire de Rheologie, INPG, UJF, CNRS (UMR 5520), 1301 rue de la piscine, BP 53 Domaine Universitaire, F-38041 Grenoble Cedex 9, France
}

Keywords:

Soft colloids

rheology of concentrated suspension

Generic

\begin{abstract}
A B S T R A C T
We extend the Maffettone-Minale model by including non-elliptical shapes of dispersed particles, a new family of internal forces controlling particle deformations, and particle-particle interactions. The last extension is made by transposing the way the chain-chain interactions are mathematically expressed in the reptation theory to suspensions. The particle-particle interactions are regarded as a confinement to cages formed by surrounding particles and by introducing a new dissipative motion (an analog of the reptation motion) inside the cages. Nonlinear responses to imposed shear and elongational flows are found to be in qualitative agreement with available experimental data.
\end{abstract}

\section{Introduction}

In this paper we are addressing rheology of colloidal dispersions (see e.g. [1,2] for a recent review). Our objective is twofold. First, we investigate relation between mesoscopic physics of dispersions and their rheological behavior. Our second objective is to illustrate the systematic formulation of governing equations of rheological models that is based on the requirement of compatibility of their solutions with mechanics (in the sense that the time reversible part of the time evolution is Hamiltonian) and with thermodynamics (in the sense that the entropy does not decrease during the time evolution).

The level of description on which we formulate the mesoscopic physics of dispersions in this paper is the level on which one symmetric three-by-three tensor (or possibly several of such tensors) serves as a state variable describing the internal structure. We call it Hand level of description since this type of the internal state variable appeared first in [3]. We shall use the symbol $\boldsymbol{c}$ to denote the tensor. After choosing $\boldsymbol{c}$ as the internal state variable, we have to decide what is its physical interpretation. Among many possibilities (see e.g. [4-9]), we choose in this paper to follow Maffettone and Minale in [10], and regard it as a mathematical representation of the ellipsoidal shape of the suspended particle. We call any rheological model on the Hand level of description with this physical interpretation of $\boldsymbol{c}$ as the Maffettone-Minale model (or in an abbreviated form MM-model).

\footnotetext{
* Corresponding author.

E-mail address: miroslav.grmela@polymtl.ca (M. Grmela).
}

What physics can we express in the MM-models? We begin with the most important physical feature. If we restrict ourselves to dispersed particles that are made of deformable but incompressible materials then the volume of the particle characterized by $c$ has to remain constant during the time evolution. If we consider $\operatorname{det} \boldsymbol{c}$ as a measure of the volume of the ellipsoid associated with $\boldsymbol{c}$ then the constraint $\operatorname{det} \boldsymbol{c}=$ const. is the first, most important, physical feature of the MM-models. Next, we have to address the question of what are the forces that control the particle deformations. In this paper we take them to be the forces generated by the surface area of the particle. We show that this type of MMmodels predicts rheological behavior that is in a good qualitative agreement with the experimental data reported in [11]. We then continue and introduce more complex physics into dispersions. In particular, we introduce: (i) more complex non-ellipsoidal shapes of the particles, (ii) particle-particle interactions, and (iii) new internal forces driving non-ellipsoidal deformations. The MM-model with these new features is called an extended MMmodel or in an abbreviated form EMM-model.

Regarding the mathematical formulation of the model, particularly new is the way in which we are expressing the particle-particle interactions. This type of interactions, that together with large particle deformations may lead to a formation of an interconnected network of particles [12], is expected to play an important role in more dense dispersions. We are inspired by the reptation theory developed by de Gennes and Edwards $[13,14]$ in which chain-chain interactions are seen as confinements to tubes (formed by surrounding chains) inside which a new dissipative motion, called reptation, takes place. In dispersions we replace tubes by cages 
(as it has been suggested on more microscopic levels of description in $[15,16]$ ) inside which a new dissipative motion (analogical to reptation) takes place.

We conclude the introduction by a comment about different levels of description. The Hand level that we are choosing in this paper is very much on the macroscopic end of the hierarchy of levels. On the other hand, the level of description chosen for example in [16]) in direct molecular simulations is on the opposite (microscopic) end. The advantage of more macroscopic theories is a large domain of applicability and a relative simplicity of the passage from governing equations to predictions that can directly be compared with experimental results. Their disadvantage is then an inevitable presence of material parameters which, due to a large "distance" from microscopic physics, are not directly related to microscopic characterizations of the particles. Given a specific colloid, their values representing it have to be found by considering some of the macroscopic observations as their measurements. The advantage of microscopic levels is the possibility to begin the analysis with a very clear and specific microscopic physics. The disadvantage is then small domain of validity (caused by the specificity of the microscopic physics entering the modeling) and the complexity of the passage to macroscopic levels on which the experimental observations are made. Such passage is inevitably involves largely ad hoc approximations needed for example to express macroscopic forces (as e.g. temperature gradient) in microscopic terms or to extract from the output of direct simulations (particle trajectories) the macroscopic information of direct interest. The modeling that has most advantages and the least disadvantages is a multilevel modeling pursued on several different levels of description.

\section{Maffettone-Minale model}

In this section we present the MM-model as a particular realization of GENERIC. The advantages of such presentation are the following: (i) the model, even before the modifications made in the subsequent section, is more general and more complete than the model introduced originally in [10], (ii) the intrinsic compatibility of the morphology time evolution with the expression for the stress tensor is guaranteed, and (iii) the extension of the MM-model needed to express more complex physics becomes straightforward (see Section 3).

First, we recall some basic elements of GENERIC. The objective of GENERIC is to make the reduction process leading from a microscopic insight to a mesoscopic model more systematic and transparent. The main idea is that before starting the reduction we make an assumption that the target mesoscopic equation possesses a certain structure. The requirement that the structure emerges in the reduction makes the reduction well organized. What is the structure of the mesoscopic time evolution equations and why it is required? We require it because it guarantees that solutions to equations possessing it agree with certain basic experimental observations like for instance conservations of mass, momentum and energy and observations constituting the experimental basis of thermodynamics. The mathematical formulation of the structure has emerged gradually. The mechanical content has been formulated in the abstract Hamiltonian structure (first for fluid mechanics equations [17] and for kinetic equations [18]) and the thermodynamic content in the structure of gradient dynamics (in [19-21]). Both structures have been then combined in [22-26]. A particular formulation of the combination (see (1) below) has been called GENERIC (an acronym for General Equation for Non-Equilibrium Reversible-Irreversible Coupling) in $[27,28]$. Geometrical and physical content of the structure has recently been discussed in [29].
In this paper we limit ourselves to suspensions that are kept at constant temperature. We recall therefore GENERIC only in this particular case. Let $x$ stand for the set of state variables. The equation governing its time evolution has the general form

$\frac{\partial x}{\partial t}=L(x) \Phi_{x}-\left[\frac{\partial \Xi}{\partial x^{*}}\right]_{x^{*}=\Phi_{x}}$

The symbol $\Phi(x)$ stands for the free energy. The first term on the right hand side of (1) represents the Hamiltonian time evolution. The operator $L(x)$, called a Poisson bivector, expresses mathematically the kinematics of $x$. The properties that $L$ is required to satisfy are best expressed by constructing with it a bracket $\{A, B\}=\left\langle A_{x}, L(x) B_{x}\right\rangle$ and requiring that it is a Poisson bracket (i.e. $\{A, B\}=-\{B, A\}$ and the Jacobi identity holds). The notation that we use in (1) and throughout the paper is the following: $A$ and $B$ are real valued sufficiently general functions of $x, A_{x}$ denotes a derivative of $A$ with respect to $x$, and $\langle$,$\rangle denotes scalar product.$ The Hamiltonian time evolution is thus an evolution generated by a potential (in the case of isothermal systems it is the free energy) in which the gradient of the potential (a covector field) is transformed into a vector field by the Poisson bivector $L$.

The second term on the right hand side represents the thermodynamic part of the time evolution. This time evolution is also generated by the free energy but the geometrical structure transforming its gradient into the vector field is different from the one playing this role in the Hamiltonian dynamics. We refer the reader who is interested in a more detailed physical and geometrical discussion of (1) to [29]. Here we only explain the notation: $\Xi\left(x, x^{*}\right)$ is the dissipation potential (see [21]), i.e. a potential satisfying: (i) $\Xi(x, 0)=0$ for all $x$; (ii) $\Xi\left(x, x^{*}\right)$ reaches its minimum as a function of $x^{*}$ for all $x$; and (iii) $\Xi\left(x, x^{*}\right)$ is a convex function of $x^{*}$ in a neighborhood of $x^{*}=0$ for all $x$. We note that these properties guarantee that $\left\langle x^{*}, \Xi_{X^{*}}\right\rangle>0$ which, together with the property $\left\langle\Phi_{x}, L(x) \Phi_{x}\right\rangle=0$, implies that $\frac{d \Phi}{d t}>0$ during the time evolution governed by (1).

We recognize in (1) four modules: Module 1 state variables $x$, Module 2 their kinematics L, Module 3 dissipation potential $\Xi$, and Module 4 free energy $\Phi$.

In the rest of this paper we formulate a mesoscopic rheological model of soft colloids. We shall do it by expressing our physical insight into this type of fluids in the four modules listed above. The essential difference between this and the more usual derivation is that in the latter the physical insight is expressed directly in one step in the governing equation while in our derivation the process is gradual, module by module. The way the modules are then combined, according to (1), guarantees agreement of solutions of the governing equations with certain results of experimental observations.

\subsection{Module 1: State variables $x$}

The point of departure of any type of rheological modeling is always the choice of the level of description, or in other words, the choice of state variables. We have to decide how shall we express our physical insight mathematically. We can choose between microscopic levels on which we can directly follow details and more macroscopic levels on which only some selected aspects of the morphology are followed. Optimally, we want to follow only those details that are essential for our interest determined by our experimental observations and intended applications. Since we cannot know in advance of what type of physics is important and what can be ignored, the choice of state variables is always a trial-and-error procedure.

In this paper we decide to remain on a rather macroscopic level of description. The advantage of this choice is a wide range of 
applicability of the resulting models and relative simplicity of the problem of solving the governing equations. The disadvantage is a rather indirect relation between our microscopic insights and modules representations that shows itself in the phenomenological nature of the material parameters entering the considerations.

We let ourselves to be inspired by the Maffetone-Minale model [10] in which the morphology of the suspension is chosen to be characterized by one conformation tensor $\boldsymbol{c}(\boldsymbol{r})$ that represents an ellipsoid. We thus regard the fluid under consideration as a suspension of droplets of ellipsoidal shapes. In fact, all the droplets are represented by one "average" droplet. Consequently, we begin with the state variables

$$
\begin{aligned}
x= & (\boldsymbol{u}(\boldsymbol{r}), \boldsymbol{c}(\boldsymbol{r})) \\
& \boldsymbol{c} \text { is a symmetric and positive definite tensor } \\
& \text { constrained by det } \boldsymbol{c}=\text { const. }
\end{aligned}
$$

By $\boldsymbol{u}(\boldsymbol{r})$ we denote the overall momentum field. The suspension is assumed to be isothermal and (overall) incompressible. Moreover, the droplets are deformable but incompressible in the sense that their volume, i.e. detc, remains a constant. We shall moreover restrict ourselves only to spatially homogeneous internal structure, i.e. we assume that $\boldsymbol{c}$ is independent of the position vector $\boldsymbol{r}$. The volume of the space region confining the suspension under consideration will be hereafter put equal to one. We recall that even if we are eventually interested in this paper only in rheological observations in which the overall momentum $\boldsymbol{u}$ is imposed and is not thus an independent state variable, we have to include it in (2). This is because the fluid described by (1) approaches thermodynamic equilibrium (a state for which the free energy reaches its minimum). It is exactly the compatibility with equilibrium that determines (as we shall see below) the admissible coupling (i.e. an expression for the stress tensor) between overall momentum and the internal structure (characterized by the tensor $\boldsymbol{c}$ ).

\subsection{Module 2: kinematics $L$}

The kinematics of (2) is well known (see e.g. [30]). It is expressed in the Poisson bracket

$$
\begin{aligned}
\{A, B\}= & \{A, B\}^{(u)}+\{A, B\}^{(c)} \\
\{A, B\}^{(u)}= & \int d \boldsymbol{r}\left[u_{i}\left(\partial_{j}\left(A_{u_{i}}\right) B_{u_{j}}-\partial_{j}\left(B_{u_{i}}\right) A_{u_{j}}\right)\right] \\
\{A, B\}^{(c)}= & +c_{k i}\left(A_{c_{i m}} \partial_{k}\left(B_{u_{m}}\right)-B_{c_{i m}} \partial_{k}\left(A_{u_{m}}\right)\right) \\
& +c_{k m}\left(A_{c_{i m}} \partial_{k}\left(B_{u_{i}}\right)-B_{c_{i m}} \partial_{k}\left(A_{u_{i}}\right)\right) \\
& -\frac{2}{3} c_{k l}\left(A_{c_{k l}} \partial_{j}\left(B_{u_{j}}\right)-B_{c_{k l}} \partial_{j}\left(A_{u_{j}}\right)\right)
\end{aligned}
$$

where $A$ and $B$ are real valued sufficiently regular functions of (2), $A_{\boldsymbol{u}(\boldsymbol{r})}$ denotes derivative of $A$ with respect to $\boldsymbol{u}(\boldsymbol{r})$, similarly $A_{\boldsymbol{c}}$ is the derivative of $A$ with respect to $\boldsymbol{c}$. We use the summation convention, and $\partial_{i}=\frac{\partial}{\partial r}$. The last line arises due to the constraint $\operatorname{det} \boldsymbol{c}=$ const (see [31])

\subsection{Module 3: dissipation potential $\Xi$}

Here arises the main technical problem of the model. How to formulate a physically meaningful dissipation term with which the time evolution obeys the constraint $\operatorname{det} \boldsymbol{c}=$ const.? The first solution to this problem has been worked out in [10] (this paper appeared in August 1998). A different solution in a different physical context, has been developed in [32] (this paper appeared in January 1999). A more general solution has been then presented in [31]. The dissipation potential formulation of the dissipative term (see the second term on the right hand side of (1)) that we use in this paper allows us to include the constraint still in a more general and a simpler way.

Following the spirit of classical nonequilibrium thermodynamics, we introduce first a thermodynamic force (we shall use the symbol $\boldsymbol{X}$ to denote it) generating the dissipation. Next, we let the dissipation potential $\Xi$ depend on $\boldsymbol{c}^{*}$ only through its dependence on $\boldsymbol{X}$, namely $\Xi\left(\boldsymbol{c}^{*}\right)=\xi\left(\boldsymbol{X}\left(\boldsymbol{c}^{*}\right)\right)$. We note that if we choose the force to be

$\boldsymbol{X}=\boldsymbol{c}^{*}-\frac{1}{3} \operatorname{tr}\left(\boldsymbol{c} \boldsymbol{c}^{*}\right) \boldsymbol{c}^{-1}$

then we see (by a direct verification) that the constraint $\operatorname{det} \boldsymbol{c}=$ const. holds (i.e. $\frac{d \operatorname{det} \boldsymbol{c}}{d \boldsymbol{c}} \Xi_{\boldsymbol{c}^{*}}=0$ ) [Indeed, note that $\frac{d \operatorname{det} \boldsymbol{c}}{d \boldsymbol{c}}=(\operatorname{det} \boldsymbol{c}) \boldsymbol{c}^{-1}$ and $\Xi_{c_{i j}^{*}}=\xi_{X_{k l}}\left(\delta_{i k} \delta_{j l}-\frac{1}{3} c_{i j} c_{k l}^{-1}\right)$ and consequently $\left.(\operatorname{det} \boldsymbol{c}) \operatorname{tr}\left(\xi_{\boldsymbol{c}^{*}}\left(\boldsymbol{c}^{-1}-\boldsymbol{c}^{-1}\right)\right)=0\right]$.

If we limit ourselves to situations that are not too far from thermodynamic equilibrium then (since the thermodynamic force (4) disappears at equilibrium) we can choose the quadratic potential

$\xi^{(c)}=\frac{1}{2} \operatorname{tr}(\boldsymbol{X} \boldsymbol{\Lambda} \boldsymbol{X})$

where $\boldsymbol{\Lambda}$ is a positive definite tensor that can depend on $\boldsymbol{c}$. We choose it to be

$\Lambda=\Lambda \boldsymbol{c}$

where $\Lambda>0$ is a scalar material parameter (recall that $\boldsymbol{c}$ is required to be positive definite - see (2) - so that $\boldsymbol{\Lambda}$ given in (6) is indeed positive definite).

\subsection{Module 4: free energy $\Phi$}

In the free energy

$\Phi(\boldsymbol{u}, \boldsymbol{c})=E(\boldsymbol{u}, \boldsymbol{c})-k_{B} T S(\boldsymbol{u}, \boldsymbol{c})$

we choose the entropy $S$ as

$S(\boldsymbol{c})=\chi \ln \operatorname{det} \mathbf{c}$

where $\chi$ is a material parameter and $k_{B}$ the Boltzmann constant. And the energy $E$ as

$E(\boldsymbol{u}, \boldsymbol{c})=E_{\text {kin }}(\boldsymbol{u})+E_{\text {pot }}(\boldsymbol{c})$

where

$E_{k i n}(\boldsymbol{u})=\int d \boldsymbol{r} \frac{\boldsymbol{u}^{2}}{2 \rho}$

and

$E_{\text {pot }}(\boldsymbol{c})=\Gamma\left((\operatorname{trc})^{2}-\operatorname{trcc}\right)$

$\rho$ and $\Gamma$ are material parameters. Since $\left((\operatorname{trc})^{2}-\operatorname{trcc}\right)$ is a measure of the area of the surface of the ellipsoid, the parameter $\Gamma$ has the physical interpretation of the surface tension. The entropy (8) is the classical Boltzmann entropy expressed in terms of $\boldsymbol{c}$ (obtained by maximizing $-\int d R f(R) \ln f(R)$ subjected to the constraint $\left.\int d R R_{i} R_{j} f(R)=c_{i j} ; R \in \mathbb{R}^{3}\right)$. The parameter $\rho$ in the kinetic energy (10) is the mass density, that, since the fluid under consideration is incompressible, is a constant. The potential energy is assumed to depend on the surface of the ellipsoid which is mathematically expressed as dependence on the second invariant of $\boldsymbol{c}$.

\subsection{Governing equations}

With the above specifications, Eq. (1) becomes

$\frac{\partial u_{i}}{\partial t}=-\partial_{j}\left(u_{i} \Phi_{u_{j}}\right)-\partial_{i} p-\partial_{j} \sigma_{i j}$ 


$$
\begin{aligned}
\frac{\partial c_{i j}}{\partial t}= & c_{k i}\left(\partial_{k} \Phi_{u_{j}}-\zeta D_{k j}\right)+c_{k j}\left(\partial_{k} \Phi_{u_{i}}-\zeta D_{i k}\right)-\frac{2}{3} c_{i j}\left(\partial_{k} \Phi_{u_{k}}\right. \\
& \left.-\zeta D_{k k}\right)-\left[\Xi_{c_{i j}^{*}}\right]_{c^{*}=\Phi_{c}}
\end{aligned}
$$

where $D_{k j}=\frac{1}{2}\left(\partial_{k} \Phi_{u_{j}}+\partial_{j} \Phi_{u_{k}}\right)$. The scalar pressure $p$ appearing in the first equation in (1) is determined by the incompressibility constraint, the extra stress tensor $\sigma$ arising in the same equation is given by

$\sigma_{i j}=-2(1-\zeta) c_{i k} \Phi_{c_{k j}}+\frac{2}{3}(1-\zeta) \delta_{i j} c_{k l} \Phi_{c_{k l}}$

If written explicitly, the derivatives of the thermodynamic and the dissipation potential appearing in (12) become

$\Phi_{\boldsymbol{u}}=\frac{\boldsymbol{u}}{\rho}$

$\Phi_{\boldsymbol{c}}=2 \Gamma \operatorname{tr}(\boldsymbol{c}) \boldsymbol{\delta}-2 \Gamma \mathbf{c}-k_{B} T \gamma \mathbf{c}^{-1}$

$\Xi_{\mathbf{c}^{*}}=\frac{1}{2}(\boldsymbol{\Lambda} \boldsymbol{X}+\boldsymbol{X} \boldsymbol{\Lambda})-\frac{1}{3} \operatorname{tr}\left(\boldsymbol{\Lambda} \boldsymbol{X} \boldsymbol{c}^{-1}\right) \boldsymbol{c}$

We easily verify directly that det $\boldsymbol{c}$ remains constant during the time evolution governed by (12). We need to show that $\frac{d \operatorname{det} \boldsymbol{c}}{d t}=(\operatorname{det} \boldsymbol{c}) \operatorname{tr}\left(\boldsymbol{c}^{-1} \frac{\mathrm{d} \boldsymbol{c}}{d t}\right)=0$. Indeed, if we multiply (15) with $\boldsymbol{c}^{-1}$ and then make trace of the result we clearly obtain zero independently of the particular choice of $\boldsymbol{\Lambda}$ and $\boldsymbol{X}$.

Summing up, the governing Eq. (1) represent (essentially if not literary) the Maffetone-Minale model introduced in [10]. The material parameters $\mathcal{P}_{M M}$ entering the MM-model are

$\mathcal{P}_{\text {MM }}=(\zeta, \chi, \Gamma, \Lambda)$

We end this section with a few remarks. The equation governing the time evolution of $\boldsymbol{u}$ that appears by inserting the specifications made in Sections 2.1,2.2,2.3,2.4 into (1) appears without the parameter $\zeta$ (i.e. Eq. (12) in which $\zeta=0$ ). Following [33], we have modified the equation by including slip into the advection of $\boldsymbol{c}$. If $\zeta=0$ then no slip occurs and the colloidal particles are passively and completely advected. If, on the other hand, $\zeta=1$ then the fluid only slides around the suspended particles and the particles themselves remain completely unaffected by the flow. From the physical point of view, the origin of the slip is a dissipative mechanism involving two velocities: one is the overall velocity $\boldsymbol{u} / \rho$ and the other is the velocity in a close neighborhood of the fluid-particle interface. Consequently, in order to include the slip directly into the setting of Eq. (1) we need to enlarge the set of state variables (2) by adopting another velocity-type field representing the local velocity close to the interface. A general physical background of this extension is discussed in [34] and the specific implementation on the Hand level of description is made in $[35,7]$.

Finally, we comment about the expression (13) for the extra stress tensor $\boldsymbol{\sigma}$. The advantage of using the modeling based on Eq. (1) is manifested in particular in the fact that the expression for the extra stress tensor emerges together with the equation governing the time evolution of $\boldsymbol{c}$. It means that in order to obtain $\boldsymbol{\sigma}$ expressed in terms of $\boldsymbol{c}$ we do not need any additional physical considerations and the expression is guaranteed to be compatible with the time evolution of $\boldsymbol{c}$. We recall that in the standard derivations of rheological models that do not use the structure (1), derivation of $\boldsymbol{\sigma}$ as a function of $\boldsymbol{c}$ requires an independent analysis and the compatibility of the time evolution of $\boldsymbol{c}$ and the expression for $\boldsymbol{\sigma}$ is not guaranteed (for example, the parameter $\zeta$ is often absent in the expression for $\boldsymbol{\sigma}$ - see more in [36]).

\section{Extended Maffettone-Minale model}

Our objective now is to adapt the Maffetone-Minale model recalled in the previous section to more concentrated hard and soft colloids. As we have already mentioned in Section 1, the new physics that we have to introduce into the model is the presence of topological interactions and the complexity of shapes of the suspended particles.

We begin with the topological interactions that we regard, in accordance with $[12,16]$, as a confinement to a cage formed by neighbor particles. There are essentially two distinct ways to express confinements in dynamics. The first is through a particular form of the potential energy (an energy landscape of traps of various depths see [37-39]) and the second through a new dissipative mechanism in an extra dimension $[13,14]$. We shall follow the latter path.

First, we recall the way the new dissipative mechanism is introduced in $[13,14]$. The physical system considered in these papers are concentrated suspensions of polymer chains or polymer melts. The confinement expressing chain-chain interactions has the form of a tube formed by neighbor polymer chains. The extra dimension is the backbone of the chain and the extra motion in this dimension (called reptation) is a diffusion (i.e. a dissipative motion). In order to formulate this physical insight mathematically, we begin with the one chain kinetic theory of dilute polymer suspensions in which the chain-chain interactions are not taken into account (see e.g. [40]). The morphological state variable used in this theory is the one chain distribution function $f(\boldsymbol{r}, R(s), t)$ where $\boldsymbol{r} \in \mathbb{R}^{3}$ is the position vector, $s \in \mathbb{R}$ is a coordinate along the backbone of the chain, $R(s) \in \mathbb{R}^{3}$ is the tangent vector to the chain at the backbone coordinate $s$, and $t$ denotes the time (we recall that in the analysis presented in [40] the continuous parameter $s$ is always discretized). De Gennes and Edwards introduce into this setting the tube confinement by replacing $f(\boldsymbol{r}, R(s), t)$ with $f(\boldsymbol{r}, R, s, t)$ and adding a new term proportional to the second derivative of $f$ with respect to $s$ to the right hand side of the equation governing the time evolution of the distribution function $f$. We can then follow this path and replace $f(\boldsymbol{r}, R, s, t)$ by its second moments $c_{i j}(\boldsymbol{r}, s, t)=\int d R R_{i} R_{j} f(\boldsymbol{r}, R, s, t)$. In such reduced formulation (worked out in [41]) the morphological state variable is a necklace of ellipsoids expressed mathematically as a one parameter family of symmetric positive definite matrices $\boldsymbol{c}(\boldsymbol{r}, s, t)$. At a given $s$ (and a given position vector $\boldsymbol{r}$ and the time $t$ ), the ellipsoid expressed mathematically by $\boldsymbol{c}(s)$ gives the direction of the vector tangent to the chain at the backbone coordinate $s$ (the direction of the principal axis of the ellipsoid) and also an additional information about its statistical distribution. We can also regard the passage from $\boldsymbol{c}(\boldsymbol{r}, t)$ to $\boldsymbol{c}(\boldsymbol{r}, s, t)$ as bringing into the consideration additional details of the microstructure that are hidden when $s$ is absent. The new microstructural details then participate in the time evolution by bringing a new dissipative mechanism.

Now we are in position to replace the tube confinement by the cage confinement. First, we reinterpret the physical meaning of the matrix c. We regard it now as in the MM-model, i.e. as a mathematical expression of an ellipsoid representing shape of suspended particles. Even before we introduce the parameter $s$, this new interpretation means, as we have seen in the previous section, that internal energy and constraints entering the morphology considerations are expressed in the second and the third invariants of $\boldsymbol{c}$ (i.e. in $\operatorname{tr}(\boldsymbol{c c})-(\operatorname{trc})^{2}$ and $\left.\operatorname{det} \boldsymbol{c}\right)$ rather than in the first invariant (i.e. $\operatorname{trc}$ ) as it is the case in the context of polymer chains. The parameter $s$ becomes now in the setting of cage confinement the coordinate inside the cage. This means that $s \in \mathbb{R}^{3}$ but if we make an assumption of spatial homogeneity and isotropy of suspensions under consideration then the cages are spheres and $s$ is the one dimensional 
radial coordinate. We note that the shape characterized by the continuous necklace $\boldsymbol{c}(s)$ is not anymore an ellipsoidal shape. It is analogical as expressing (in the Fourier series) a non sinusoidal function through a one parameter family of sinusoidal functions. In this paper we shall still simplify the formulation. We replace $\boldsymbol{c}(s, t)$ by $(\boldsymbol{c}(t), \boldsymbol{b}(t))$, i.e. we discretize $s$ and keep only two terms. The necklace characterizing the shapes of the suspended colloidal particles is now discrete, composed of only two beads.

In this particular case we can regard the physical picture also as follows. In order to express the collective nature of the suspended particles, we equip the representative particle that we follow in our mathematical formulation with an additional structure. The particle acquires two flavors (we borrow this terminology from the theory of elementary particles): the flavor $C$ (described by the tensor $\boldsymbol{c}$ ) and the flavor $B$ (described by the tensor $\boldsymbol{b}$ ). We can even think of the suspension as being literary composed of two types of particles: particles $C$ and particles $B$. The passing from simple (flavorless) particles to particles with two flavors does not change the volume of the particles since detc + detb is required to remain a constant. The imaginary separation into two flavors is made in order to be able to express the collectivity of the particles. The more are the flavors different from each other the more is the shape of the particle their represent different from an ellipsoid.
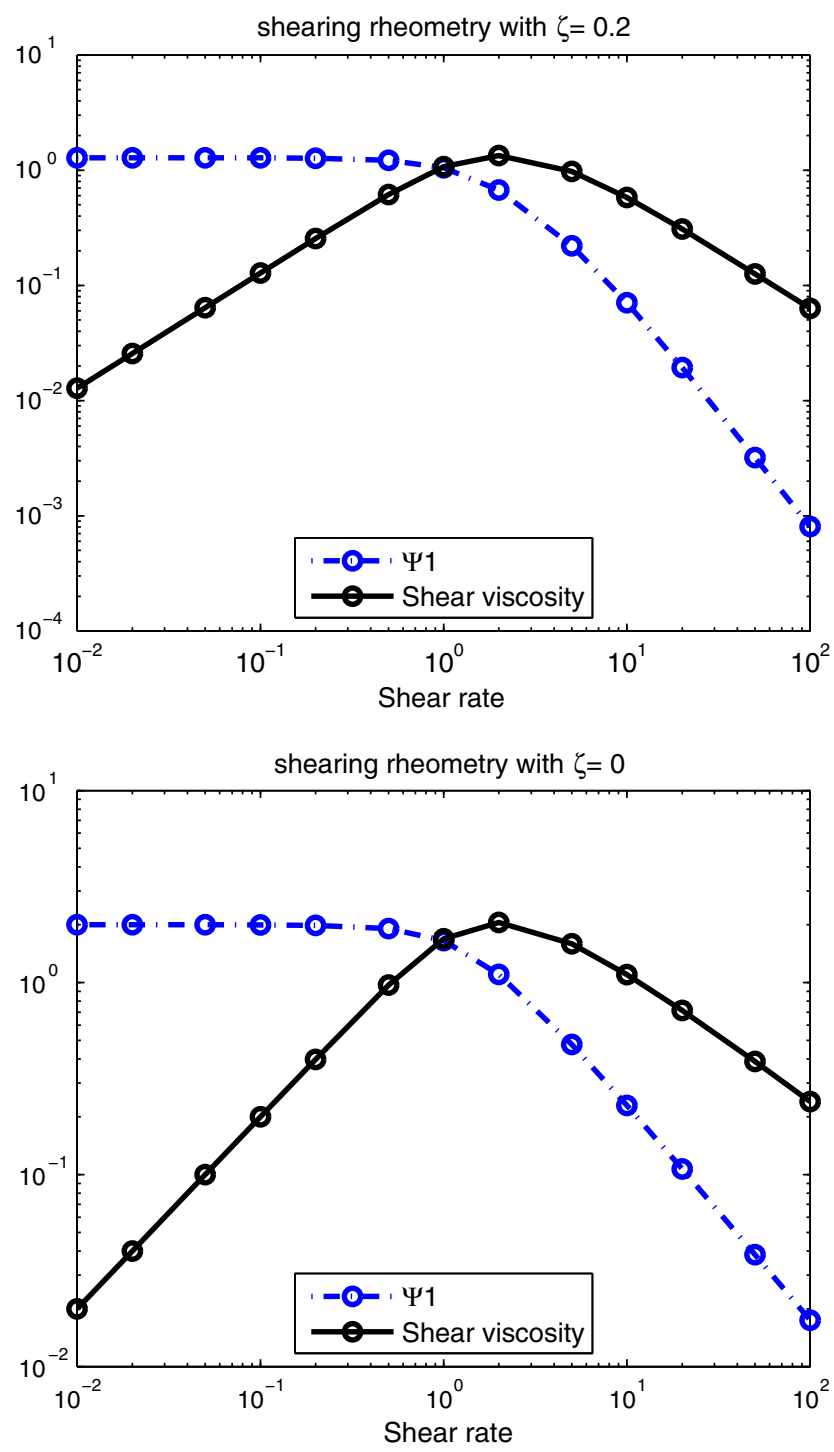

Fig. 1. Influence of the parameter $\zeta$ on the steady shear viscosity and the first normal stress difference.
Now, due to the influence of the neighbor particles, the two flavors interact with each other. First, there is an interaction expressed in the free energy (appearing in the governing equations of the model developed below in terms that proportional to the parameter $\gamma$ ) and second, there is a dissipative mechanism driving the flavors to become identical (appearing in the governing equations in terms proportional to the parameter $\lambda$ ). The first interaction brings an internal structure to the particles (expressing for example the formation or break-up of an interconnected network), the dissipative mechanism is analogical to the dissipative mechanism (reptation) in the tube constraint.

We now follow the steps in the previous section and formulate the time evolution equations.

\subsection{Module 1: State variables $x$}

With the state variables (2) we are limited to only ellipsoidal shapes. How can we reach beyond ellipsoids while still keeping a relative simplicity of the model? An obvious answer is that we shall characterize the shape of a single colloidal particle not by one ellipsoid (i.e. one tensor $\boldsymbol{c}$ ) but by two or more ellipsoids (i.e. two or more tensors $\boldsymbol{c}$ ). This way of dealing with nonellipsoidal shapes has already been used in [42] in the context of colloids in the presence of surfactants. In this paper we shall not use the "continuous necklace" representation employed in [42] (the necklace consists of a one parameter family of ellipsoids) but a "discrete necklace" representation in which the necklace consists of a finite number ellipsoids expressed mathematically by a finite number of conformation tensors. In this paper we limit ourselves to two ellipsoids on the necklace. We denote the conformation tensors representing them by the symbols $\boldsymbol{c}$ and $\boldsymbol{b}$. We are choosing this simplified representation in order to keep the problem of solving the governing equations simple.

The state variables replacing (2) are thus

$$
\begin{aligned}
x= & (\rho(\boldsymbol{r}), \boldsymbol{u}(\boldsymbol{r}), \boldsymbol{c}(\boldsymbol{r}), \boldsymbol{b}(\boldsymbol{r})) \\
& \text { both } \boldsymbol{c} \text { and } \boldsymbol{b} \text { are symmetric and positive definite } \\
& \text { constrained by } \operatorname{det} \boldsymbol{c}+\operatorname{det} \boldsymbol{b}=\text { const. }
\end{aligned}
$$

The incompressibility of the suspended particles is now mathematically expressed by $\operatorname{det} \boldsymbol{c}+\operatorname{det} \boldsymbol{b}=$ const.

\subsection{Module 2: kinematics $L$}

The Poisson bracket (3) expressing kinematics of (17) is the bracket

$\{A, B\}=\{A, B\}^{(u)}+\{A, B\}^{(c)}+\{A, B\}^{(b)}$

where $\{A, B\}^{(b)}$ is the same as $\{A, B\}^{(c)}$ except that the tensor $\boldsymbol{c}$ in it is replaced by the tensor $\boldsymbol{b}$. We shall use hereafter the superscript $(c)$ respectively $(b)$ to denote quantities associated with the presence of $\boldsymbol{c}$ respectively $\boldsymbol{b}$.

\subsection{Module 3: dissipation potential $\Xi$}

The enlarged set of state variables (17) enables us to express complex particle-particle interactions taking place in soft colloids. We shall do it by putting new terms into both the dissipation and the thermodynamic potentials. We begin with the dissipation potential. In addition to two thermodynamic forces (4) (one for $\boldsymbol{c}$ and the other for $\boldsymbol{b}$ ), we also introduce a new force, we shall denote it by the symbol $\boldsymbol{Y}$, expressing interactions in dissipative processes. The physical consideration behind the new term is the following. The thermodynamic forces $\boldsymbol{X}^{(c)}$ and $\boldsymbol{X}^{(b)}$ drive the morphology of the suspension to equilibrium at which these two thermodynamic forces disappear (and thus the suspended particles become 
spheres, i.e. $\boldsymbol{c}=\boldsymbol{b}=$ const. $\boldsymbol{\delta})$. We now introduce another force $\boldsymbol{Y}$ that drives nonellipsoidal shapes to ellipsoids (i.e. $\boldsymbol{b}=\boldsymbol{c}$ ). This force generates the diffusion along the backbone of the discrete necklace. The relaxation to spherical shapes is proceeding in two stages. One, driven by the force $\boldsymbol{Y}$, takes nonellipsoidal shapes into ellipsoidal shapes (for which $\boldsymbol{b}=\boldsymbol{c}$ ) and the second, driven by $\boldsymbol{X}^{(c)}$ and $\boldsymbol{X}^{(b)}$, takes the ellipsoids into spheres (i.e. $\boldsymbol{c}=\boldsymbol{b}=$ const. $\boldsymbol{\delta}$ ).

The forces $\boldsymbol{X}^{(c)}, \boldsymbol{X}^{(b)}$ and $\boldsymbol{Y}$ are given by

$\boldsymbol{X}^{(c)}=\boldsymbol{c}^{*}-\frac{1}{3} \operatorname{tr}\left(\boldsymbol{c} \boldsymbol{c}^{*}\right) \boldsymbol{c}^{-1}$

$\boldsymbol{X}^{(b)}=\boldsymbol{b}^{*}-\frac{1}{3} \operatorname{tr}\left(\boldsymbol{b} \boldsymbol{b}^{*}\right) \boldsymbol{b}^{-1}$

$\boldsymbol{Y}=\left(\boldsymbol{c}^{*}-\boldsymbol{b}^{*}\right)+\frac{1}{3}\left(\frac{\operatorname{det} \boldsymbol{b}}{\operatorname{det} \boldsymbol{c}}\right) \operatorname{tr}\left(\boldsymbol{c c} \boldsymbol{c}^{*}\right) \boldsymbol{b}^{-1}-\frac{1}{3}\left(\frac{\operatorname{det} \boldsymbol{c}}{\operatorname{det} \boldsymbol{b}}\right) \operatorname{tr}\left(\boldsymbol{b} \boldsymbol{b}^{*}\right) \boldsymbol{c}^{-1}$

The thermodynamic potential replacing (5) becomes

$\xi=\frac{1}{2}\left[\operatorname{tr}\left(\boldsymbol{X}^{(c)} \boldsymbol{\Lambda}^{(c)} \boldsymbol{X}^{(c)}\right)+\operatorname{tr}\left(\boldsymbol{X}^{(b)} \boldsymbol{\Lambda}^{(b)} \boldsymbol{X}^{(b)}\right)+\operatorname{tr}(\boldsymbol{Y} \lambda \boldsymbol{Y})\right]$

where $\Lambda^{(c)}, \Lambda^{(b)}$ and $\lambda$ are material parameters. It is easy to verify that this dissipation potential satisfies all the properties listed in the text that follows Eq. (1) provided $\Lambda^{(c)}, \Lambda^{(b)}$ and $\lambda$ are positive definite matrices. Moreover, we easily verify that indeed $\operatorname{det} \boldsymbol{c}+\operatorname{det} \boldsymbol{b}=$ const. remains unchanged during the time evolution (we need to show that $(\operatorname{det} \boldsymbol{c}) \boldsymbol{c}^{-1} \Xi_{\boldsymbol{c}^{*}}+(\operatorname{det} \boldsymbol{b}) \boldsymbol{b}^{-1} \Xi_{\boldsymbol{b}^{*}}=0$ ).

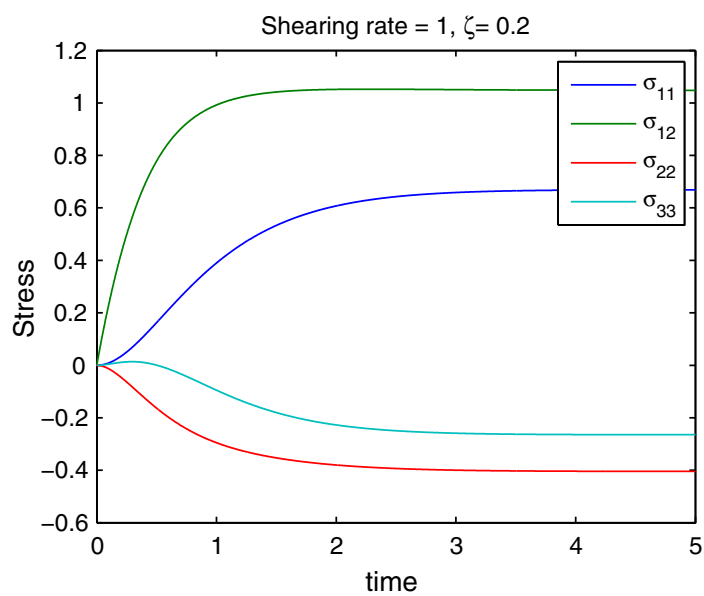

\subsection{Module 4: free energy $\Phi$}

The second place where we put the particle-particle interactions is the thermodynamic potential $\Phi$. The potential energy (11) is extended to

$$
\begin{aligned}
E_{p o t}(x)= & {\left[\Gamma^{(c)}\left((\operatorname{trc})^{2}-\operatorname{trcc}\right)+\Gamma^{(b)}\left((\operatorname{tr} \boldsymbol{b})^{2}-\operatorname{tr} \boldsymbol{b} \boldsymbol{b}\right)\right.} \\
& +\gamma \operatorname{tr}((\boldsymbol{c}-\boldsymbol{b})(\boldsymbol{c}-\boldsymbol{b}))]
\end{aligned}
$$

The third term on the right hand side is a new term. It expresses the $\boldsymbol{c} \rightleftharpoons \boldsymbol{b}$ interactions with minimum at $\boldsymbol{c}=\boldsymbol{b}$ (i.e. at colloidal particles with an ellipsoidal shape). In the formulation with the continuous necklace, this term would have the Cahn-Hilliard type from $\gamma \operatorname{tr}\left(\frac{d c}{d s} \frac{d c}{d s}\right)$. The parameters $\Gamma^{(c)}, \Gamma^{(b)}$ and $\gamma$ are material parameters. The entropy (8) is extended into

$S(x)=\chi(\ln \operatorname{det} \mathbf{c}+\ln \operatorname{det} \mathbf{b})$

and the kinetic energy (10) remains unchanged.

\subsection{Governing equations}

With the new modules, Eq. (1) becomes

$$
\begin{aligned}
\frac{\partial u_{i}}{\partial t}= & -\partial_{j}\left(u_{i} \Phi_{u_{j}}\right)-\partial_{i} p-\partial_{j} \sigma_{i j} \\
\frac{\partial c_{i j}}{\partial t}= & c_{k i}\left(\partial_{k} \Phi_{u_{j}}-\zeta^{(c)} D_{k j}\right)+c_{k j}\left(\partial_{k} \Phi_{u_{i}}-\zeta^{(c)} D_{i k}\right) \\
& -\frac{2}{3} c_{i j}\left(\partial_{k} \Phi_{u_{k}}-\zeta^{(c)} D_{k k}\right)-\left[\Xi_{c_{i j}^{*}}\right]_{c^{*}=\Phi_{\boldsymbol{c}}}
\end{aligned}
$$
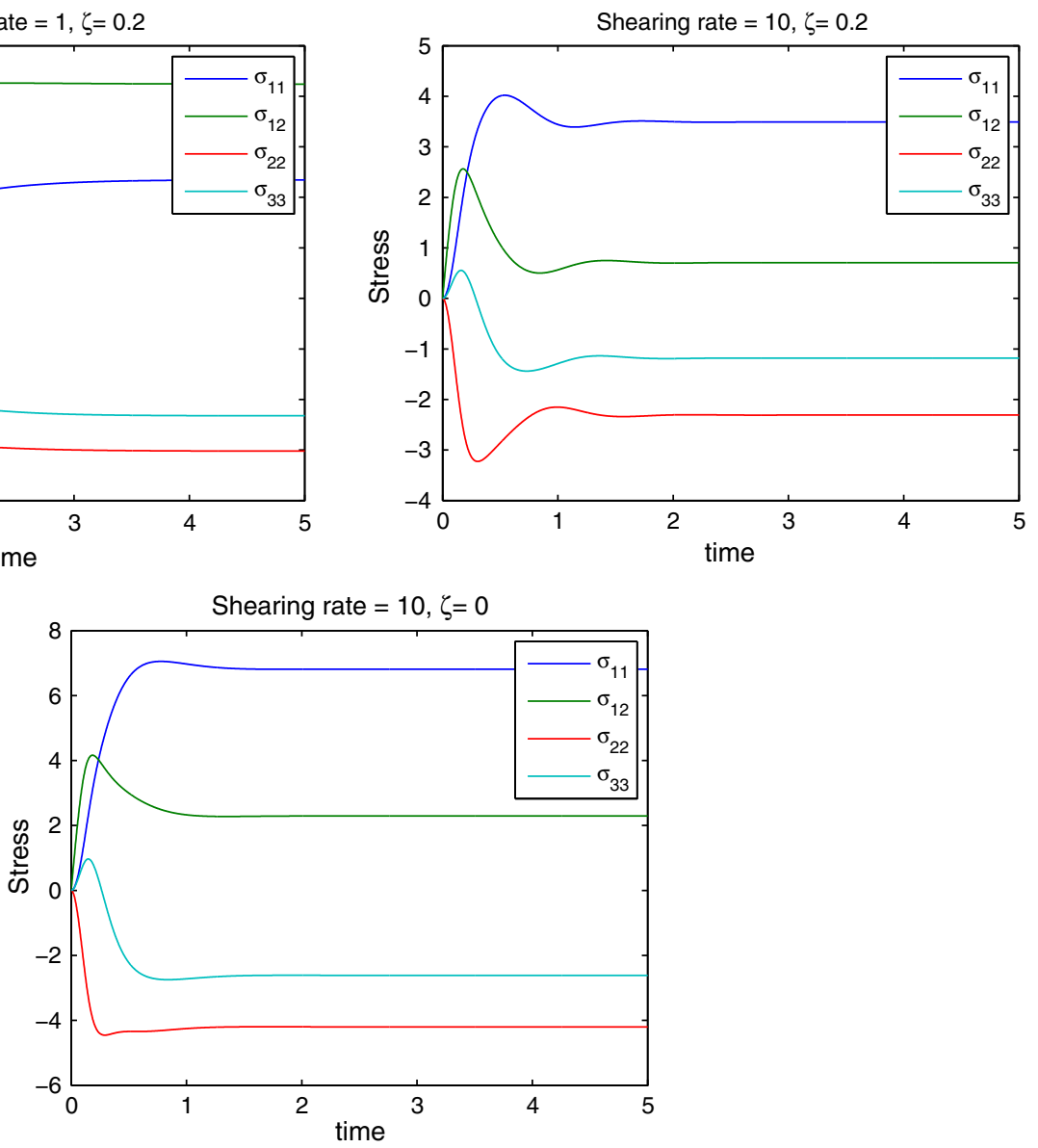

Fig. 2. Influence of the parameter $\zeta$ and the shearing rate on the start-up time evolution of the stress tensor. 


$$
\begin{aligned}
\frac{\partial b_{i j}}{\partial t}= & b_{k i}\left(\partial_{k} \Phi_{u_{j}}-\zeta^{(b)} D_{k j}\right)+b_{k j}\left(\partial_{k} \Phi_{u_{i}}-\zeta^{(b)} D_{i k}\right)-\frac{2}{3} b_{i j}\left(\partial_{k} \Phi_{u_{k}}\right. \\
& \left.-\zeta^{(b)} D_{k k}\right)-\left[\Xi_{b_{i j}^{*}}\right]_{\boldsymbol{b}^{*}=\Phi_{\boldsymbol{b}}}
\end{aligned}
$$

with

$$
\begin{aligned}
\sigma_{i j}= & -2\left(1-\zeta^{(c)}\right) c_{i k} \Phi_{c_{k j}}+\frac{2}{3}\left(1-\zeta^{(c)}\right) \delta_{i j} c_{k l} \Phi_{c_{k l}}-2\left(1-\zeta^{(b)}\right) b_{i k} \Phi_{b_{k j}} \\
& +\frac{2}{3}\left(1-\zeta^{(b)}\right) \delta_{i j} b_{k l} \Phi_{b_{k l}}
\end{aligned}
$$

The derivatives of the potentials $\Phi$ and $\Xi$ appearing in these equations have the following explicit form:

$$
\begin{aligned}
\Phi_{\boldsymbol{c}}= & 2 \Gamma^{(c)} \operatorname{tr}(\boldsymbol{c}) \boldsymbol{\delta}-2 \Gamma^{(c)} \boldsymbol{c}+2 \gamma(\boldsymbol{c}-\boldsymbol{b})-k_{B} T \chi \boldsymbol{c}^{-1} \\
\Phi_{\boldsymbol{b}}= & 2 \Gamma^{(b)} \operatorname{tr}(\boldsymbol{b}) \boldsymbol{\delta}-2 \Gamma^{(b)} \boldsymbol{b}-2 \gamma(\boldsymbol{c}-\boldsymbol{b})-k_{B} T \chi \boldsymbol{b}^{-1} \\
\Xi_{c_{i j}^{*}}= & \frac{1}{2}\left(\boldsymbol{\Lambda}^{(c)} \boldsymbol{X}^{(c)}+\boldsymbol{X}^{(c)} \boldsymbol{\Lambda}^{(c)}\right)_{i j}-\frac{1}{3} \operatorname{tr}\left(\boldsymbol{\Lambda}^{(c)} \boldsymbol{X}^{(c)} \boldsymbol{c}^{-1}\right) c_{i j} \\
& +\frac{1}{2}(\lambda \boldsymbol{Y}+\boldsymbol{Y} \lambda)_{i j}+\frac{1}{3} \operatorname{tr}\left(\lambda \boldsymbol{Y} \boldsymbol{b}^{-1}\right)\left(\frac{\operatorname{det} \boldsymbol{b}}{\operatorname{det} \boldsymbol{c}}\right) c_{i j} \\
\Xi_{b_{i j}^{*}=} & \frac{1}{2}\left(\boldsymbol{\Lambda}^{(b)} \boldsymbol{X}^{(b)}+\boldsymbol{X}^{(b)} \boldsymbol{\Lambda}^{(b)}\right)_{i j}-\frac{1}{3} \operatorname{tr}\left(\boldsymbol{\Lambda} \boldsymbol{X}^{(b)} \boldsymbol{b}^{-1}\right) b_{i j} \\
& -\frac{1}{2}(\lambda \boldsymbol{Y}+\boldsymbol{Y} \lambda)_{i j}-\frac{1}{3} \operatorname{tr}\left(\lambda \boldsymbol{Y} \boldsymbol{c}^{-1}\right)\left(\frac{\operatorname{det} \boldsymbol{c}}{\operatorname{det} \boldsymbol{b}}\right) b_{i j}
\end{aligned}
$$

Analogically to the choice of the parameter $\boldsymbol{\Lambda}$ entering the MM-model (see (6)), we choose
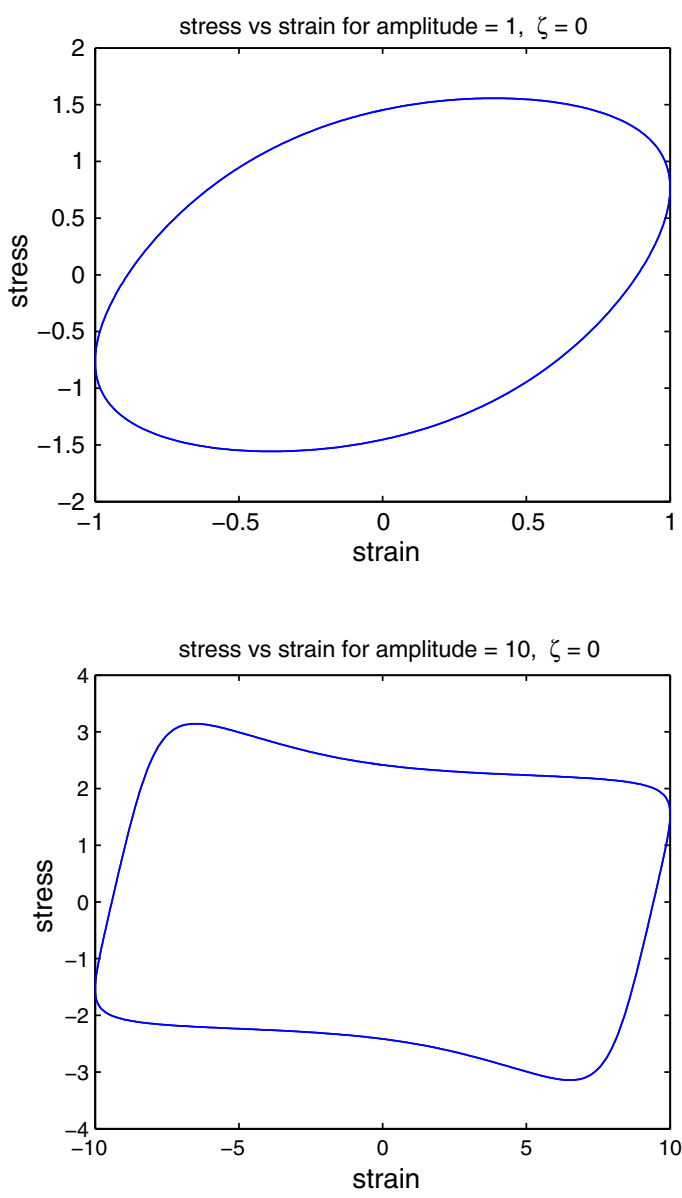

$\Lambda^{(c)}=\Lambda^{(c)} \boldsymbol{c}$

$\boldsymbol{\Lambda}^{(b)}=\Lambda^{(b)} \boldsymbol{b}$

$\lambda=\lambda(\boldsymbol{c}+\boldsymbol{b})$

where $\Lambda^{(c)}>0, \Lambda^{(b)}>0$ and $\lambda>0$ are material parameters.

Altogether, the material parameters $\mathcal{P}_{E M M}$ entering the governing Eq. (23) of the Extended Maffettone-Minale model (EMMmodel) are

$\mathcal{P}_{E M M}=\left(\Gamma^{(c)}, \Gamma^{(b)}, \gamma, \Lambda^{(c)}, \Lambda^{(b)}, \lambda, \zeta^{(c)}, \zeta^{(b)}, \chi\right)$

We note that if $\gamma=0$ and $\lambda=0$ then the EMM-model reduces to the MM-model presented in Section 2. The extension of the MM-model made in this paper consists in representing the suspended particle by a necklace of ellipsoids (in this paper we restrict ourselves to the necklace consisting of only two ellipsoids) rather than a single ellipsoid and in bringing into the model an internal interaction (expressed in the terms proportional to $\gamma$ ) and a new diffusion-type motion along the backbone of the necklace (expressed in the terms proportional to $\lambda$ ).

\section{Results}

In order to calculate rheological and morphological predictions of the models presented above, we have to make the following steps: (i) we have to specify the values of the parameters ((16) in the case of MM-model and (29) in the case of EMM-model), (ii) we have to specify the externally imposed flow (in the illustrations presented below we limit ourselves only to a simple shear flow
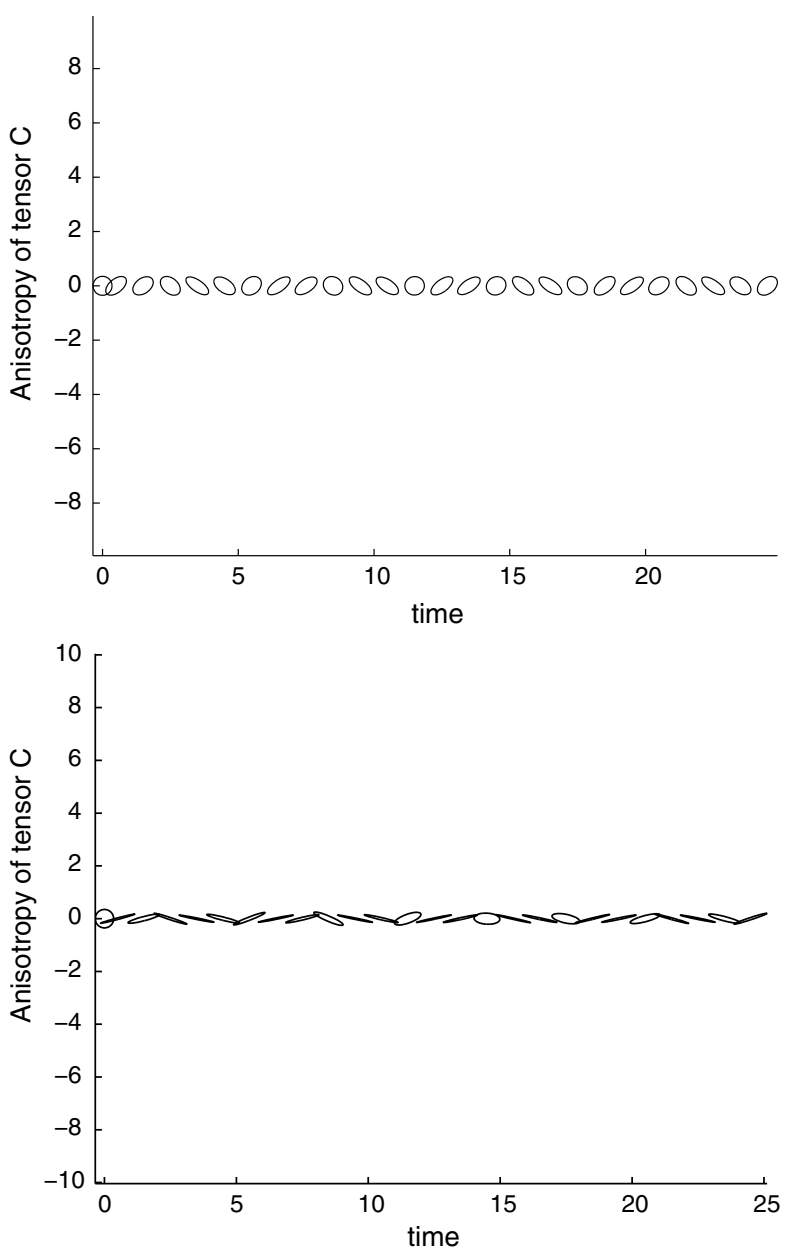

Fig. 3. Lissajous curves for $\zeta=0$. 

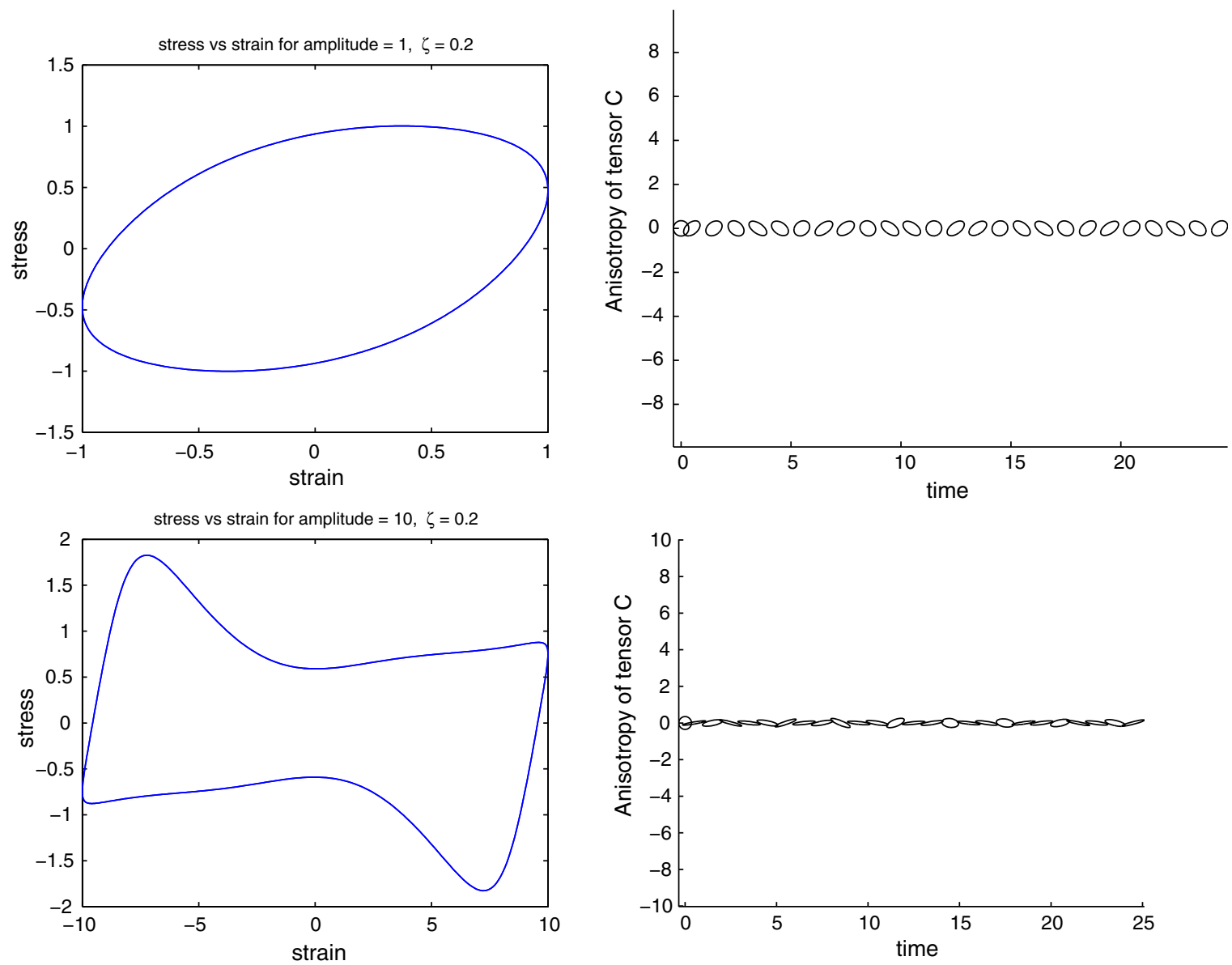

Fig. 4. Lissajous curves for $\zeta=0.2$.

that is either switched on at the time $t=0$ or changes periodically in time), (iii) we have solve the governing equations (the second equation in (12) in the case of the MM-model and the second equation in (23) in the case of the EMM-model), and (iv) we have to insert the solution to the expression for the stress tensor ((13) in the case of MM-model and (24) in the case of EMM-model). We note that the third step is in principle simple since, from the mathematical point of view, it is a problem of solving numerically a system of ordinary differential equations. We can therefore calculate relatively easily any type of rheological predictions and we can even think of implementing the model into calculations of flows taking place in processing operations.

What are the experimental observations in which the mesoscopic physics that we have expressed in the model developed in this paper may manifest itself? Such observations are certainly not the observations of linear responses to imposed oscillatory flows expressed in the coefficients $G^{\prime}$ and $G^{\prime \prime}$. This is because the physical processes that mainly influence the outcome of such observations take place on more microscopic level than the level on which our model is formulated. We anticipate that the most relevant to our model are nonlinear responses to imposed shear and elongational flows. The main source of experimental data of this type with which we are comparing our predictions is Ref. [11].

When making comparison of experimental data with model predictions we are establishing relation between two sets of parameters: $\mathcal{P}_{\text {exp }}$ and $\mathcal{P}_{\text {mod }}$. The former is composed of the parameters used in experimental observations to characterize the fluids under investigation, the latter are the parameters used for this purpose in the model. In the MM-model they are (16), in the
EMM-model they are (29). In this paper we shall remain only with a qualitative comparison of experimental and theoretical results and we shall not enter into the investigation of the relation $\mathcal{P}_{\text {exp }} \leftrightarrow \mathcal{P}_{\text {mod }}$. The more detailed quantitative comparison requires further extensions of the MM-type models in which more details of the complex physics taking place in colloids is expressed. The qualitative agreement (demonstrated below) that we find already with MM and EMM models indicates the pertinence of the mesoscopic physics that we put into these simple models. We hope to build a family of MM-models with varying amount of details of the physics involved in future papers.

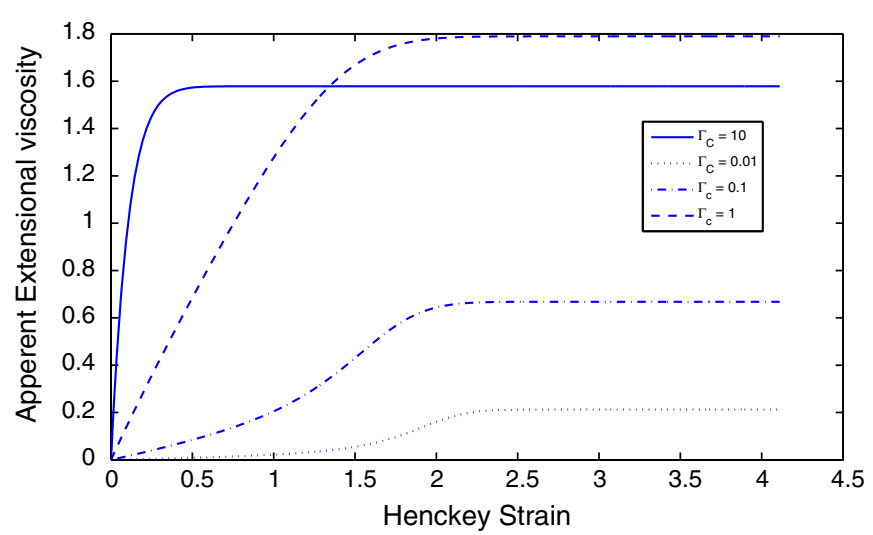

Fig. 5. Elongational response of the MM-model. 
4.1. Rheological and morphological predictions of the MM-model (12) and (13)

We recall that the mesoscopic physics that we have put into the MM-model can be summarized as follows: (i) the droplets have an ellipsoidal shape, (ii) the droplets are deformable but incompress-
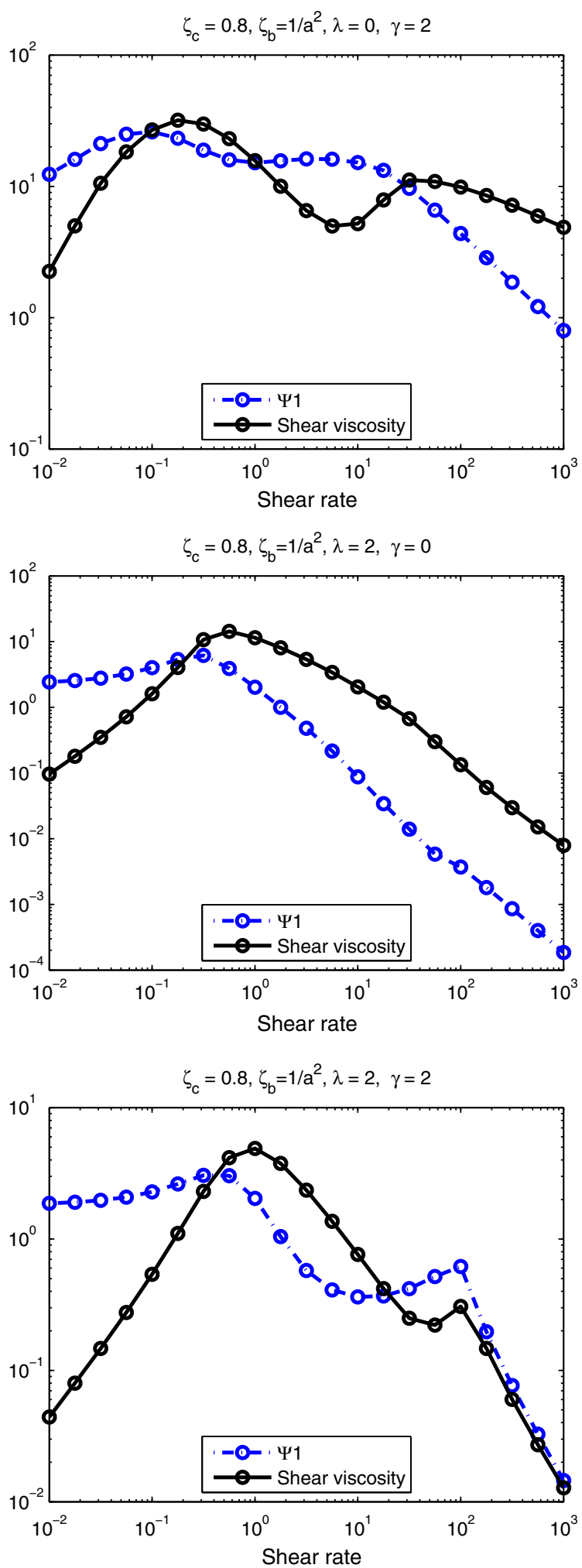

Fig. 6. Influence of $\lambda$ and $\gamma$ on the rheometry response. ible, and (iii) the droplet deformations are controlled by their surface area.

Our objective is in this section twofold. First, we show that the physics expressed in the point (i)-(iii) above is enough to reproduce the important qualitative features of the rheology of colloids, namely the appearance of shear thickening followed by shear thinning in shear viscosity versus shear rate curves (see [11]). Second, we then show what are the corresponding predictions of the behavior of the first normal stress difference, predictions of responses in elongational flows (also measured in [11]), and Lissajous curves.

For the illustration we choose the following MM-model parameters: $\Gamma_{c}=1, \Omega_{c}=1, \chi=1$ and we use two values 0 and 0.2 of $\zeta$.

Fig. 1 depicts steady shear viscosity and the first normal stress difference $\Psi_{1}$ for two values of the parameter $\zeta$. In Fig. 2 we show the start-up time evolution of the shear stress for the shear rates 1 and the parameter $\zeta=0.2$ and for the shear rate 10 and the parameter $\zeta=0.2$ and $\zeta=0$. In Figs. 3 and 4 we show the Lissajous curves for the two values of $\zeta$. The value of pulsation is fixed to 1 and the amplitude takes two values 1 and 10 . The morphology characterized by the tensor $\boldsymbol{c}$ is shown for 4 cycles of the alternate shearing starting from the rest. Fig. 5 depicts the elongational response of the MM-model. The parameters used are $\Omega_{c}=1, \chi=1, \zeta=0.5$. In order to see the indirect effet of concentration the parmeter $\Gamma_{C}$ has been modified. Differents values has been used : $0.01,0.1,1,10$. The velocity gradient tensor has a diagonal form $\partial_{1} u_{1}=2, \partial_{2} u_{2}=-1, \partial_{3} u_{3}=-1$.
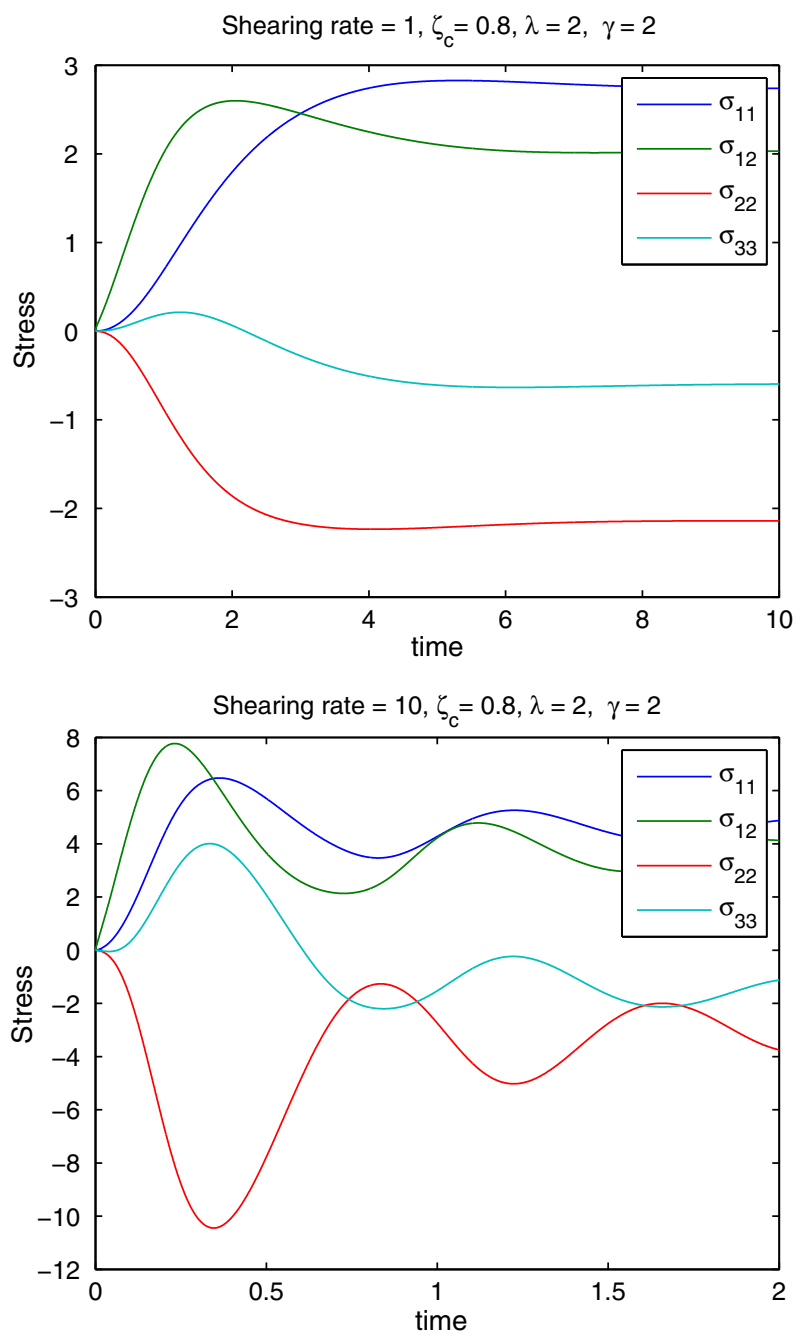

Fig. 7. Effect of shear rate on the start-up response with $\lambda=2$ and $\gamma=2$. 

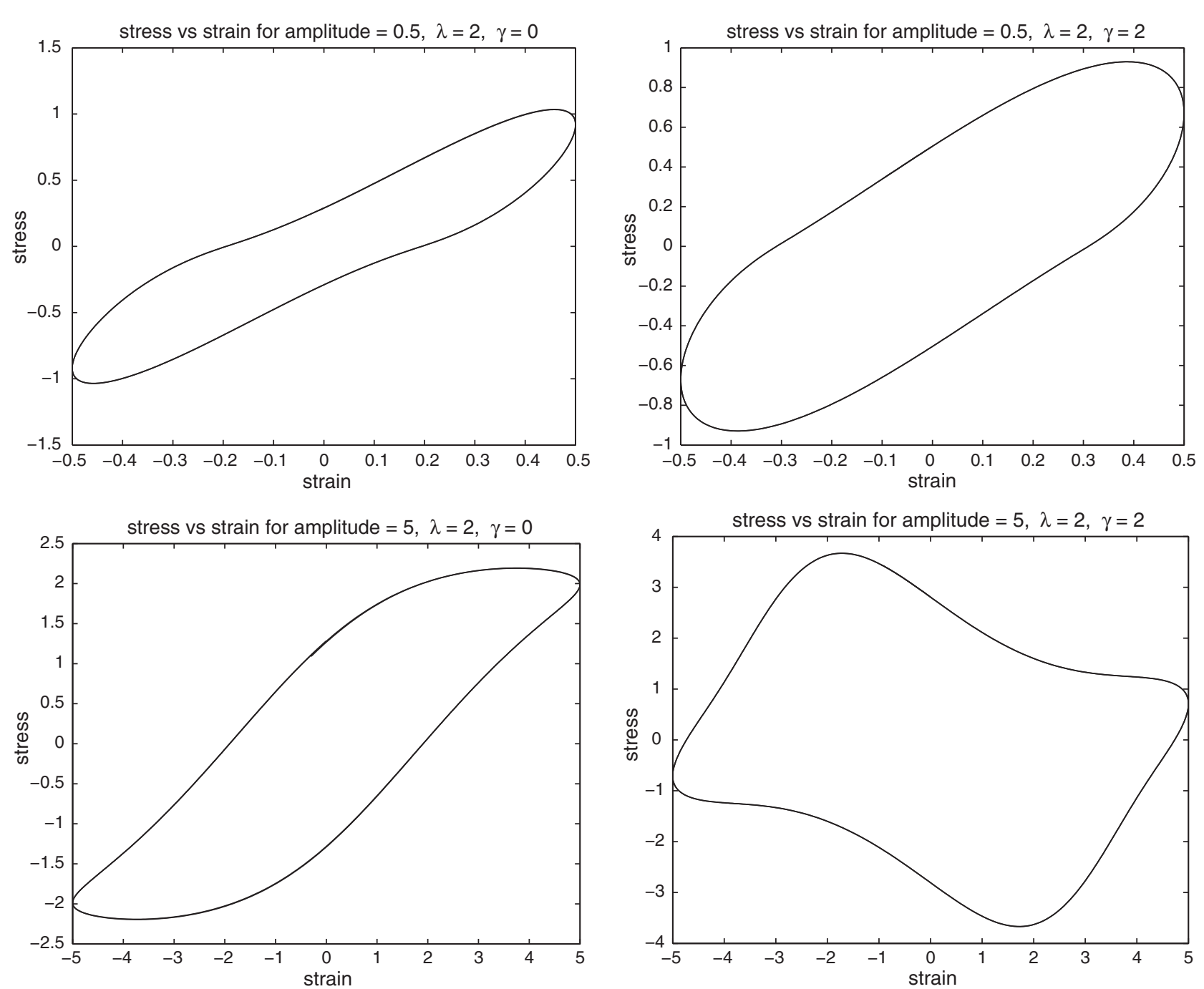

Fig. 8. Lissajous curves for $\lambda=2$ and $\gamma=0$ or 2 .

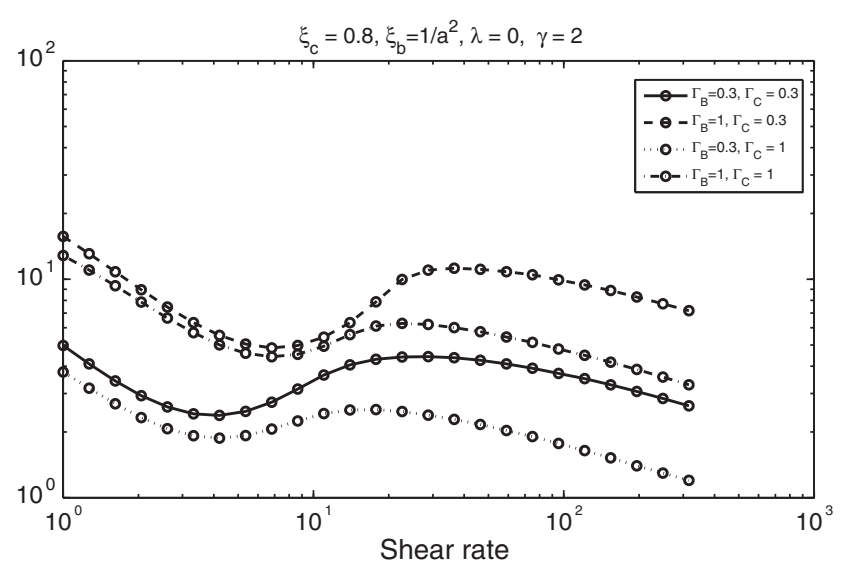

Fig. 9. Effect of concentration on the shear response of the EMM-model.

4.2. Rheological and morphological predictions of the EMM-model (23) and (24)

We recall that the EMM-model extends the MM-model by, first, allowing nonelliptical shapes of the droplets, and second, introducing new interactions, both inside the droplet and among the

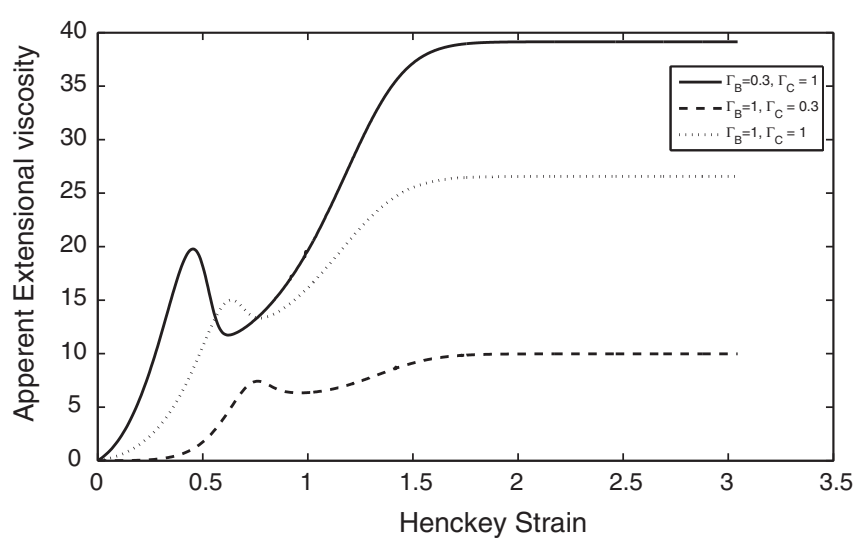

Fig. 10. Effect of concentration on the elongation response of the EMM-model.

droplets. Our objective in this section is to show how this additional physics modifies the rheological predictions.

In the EMM-model both $\boldsymbol{c}$ and $\boldsymbol{b}$ characterize the morphology. As an illustration, we calculate predictions for the parameters $\Gamma_{c}=1, \Omega_{c}=1, \chi=1, \Gamma_{b}=0.3, \Omega_{b}=0.1, \zeta_{c}=0.8, \zeta_{b}=1 / a^{2} \quad$ where $a$ is the shape factor of tensor $\boldsymbol{b}$. In Fig. 6 we plot the steady shear viscosity and the first normal stress difference curves for different 

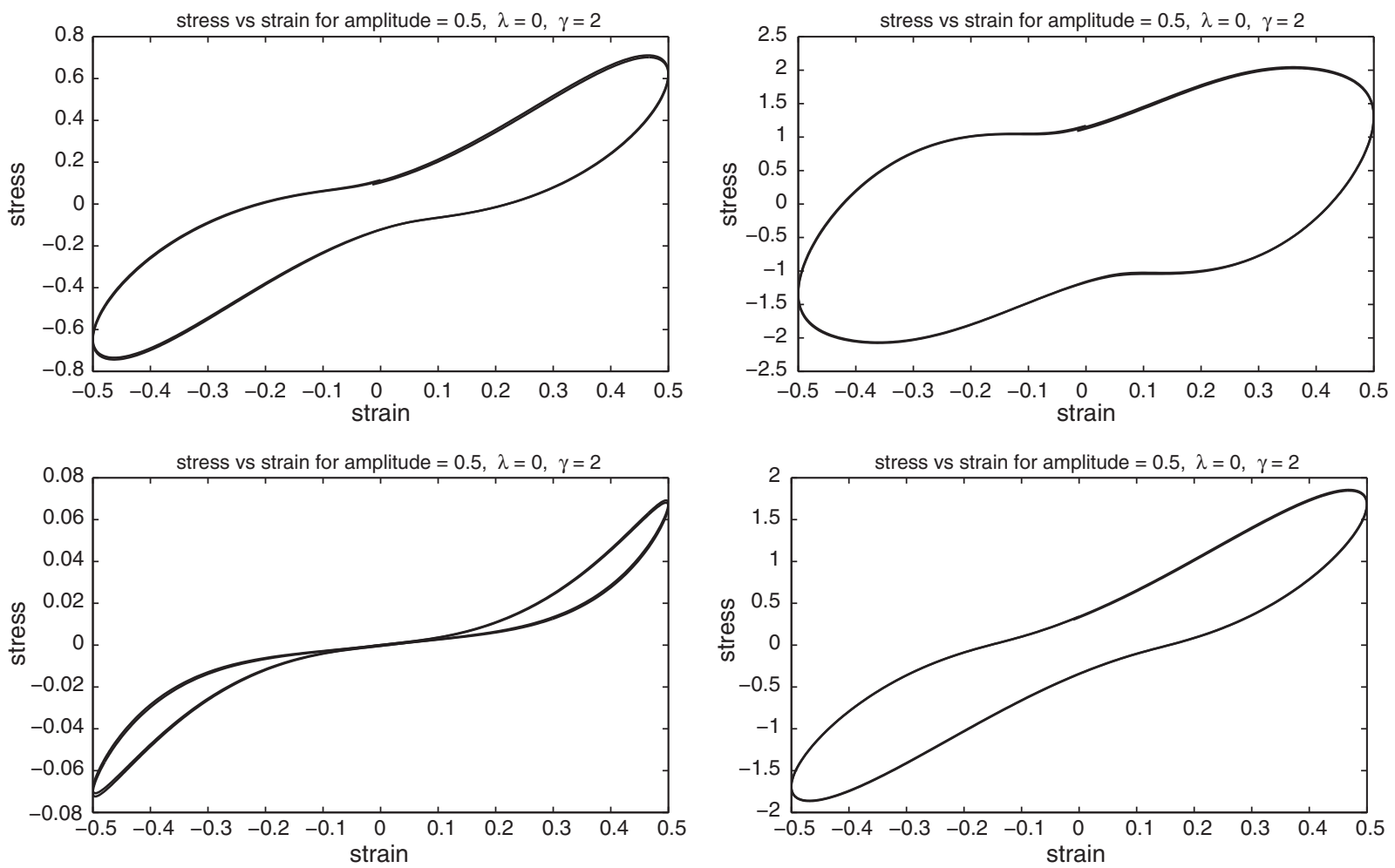

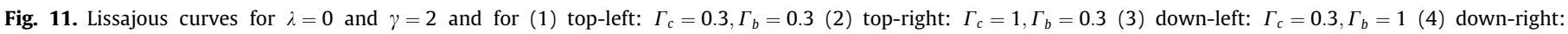
$\Gamma_{c}=1, \Gamma_{b}=1$.

values of the parameters $\lambda$ and $\gamma$. Start-up time evolution is presented in Fig. 7 for increasing shear rate : 1,10,100. Comparison of Lissajous curves with unit pulsation and increasing amplitude : $0.5,5,50$ is shown in Fig. 8 .

In order to study the effect of concentration we have fixed the following parameters: $\Omega_{c}=1, \chi=1, \Omega_{b}=0.1, \zeta_{c}=0.8, \zeta_{b}=1 / a^{2}$, $\lambda=0, \gamma=2$ and we have modified the values of parameters $\Gamma_{c}$ and $\Gamma_{b}$. This choice gives a qualitative fit to figures presented in [11]. Fig. 9 shows the effect of the modification of these last two parameters. In Fig. 10 the elongational response is shown with the velocity gradient given by the diagonal matrice $\partial_{1} u_{1}=2, \partial_{2} u_{2}=-1, \partial_{3} u_{3}=-1$.

Fig. 11 depicts the Lissajous curves for different values of $\Gamma_{c}$ and $\Gamma_{b}$ and for the frequency that equal to one.

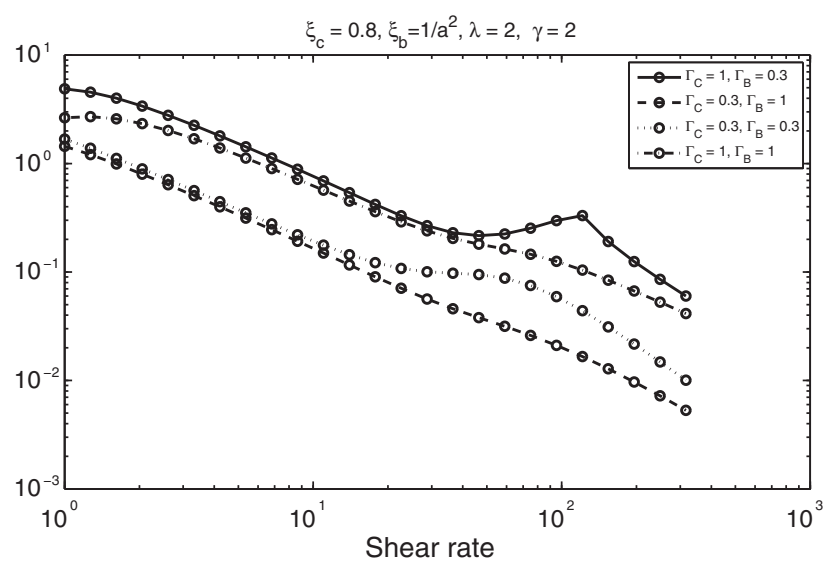

Fig. 12. Effect of the parameter $\lambda$ on the shear response of the EMM-model.
Finally, and in order the see the effect of the parameter $\lambda$, Fig. 12 shows the effect of the modification of this parameter in the interval from 0 to 2 . All the other parameters are kept without modification.

The effect of the extra term in the energy that expresses collective interactions is seen in the dependence of the predictions on the parameter $\gamma$. The emergence of the second bump on Fig. 7 can be interpreted physically as an emergence and subsequent disappearance of a collective structure (as e.g. a network structure). Indeed, we note that when $\gamma=0$ then the second bump disappears. The effect of the new dissipative mechanism (that is analogical to reptation in the context of polymer chains) is seen in the dependence of the predictions on the parameter $\lambda$. As for the role of the slip, we see again, as we have already seen in the MM-model, that the interactions, both internal and collective, become more influential with the slip.

\section{Concluding remarks}

How to model (on a mesoscopic level of description and in a relatively simple way) rheological behavior and morphology of concentrated suspensions in which particle-particle interaction play an important role? Following example of the reptation theory, we make the following two steps. First, we regard the particle-particle interactions as a confinement (to a tube in the case of polymer chains and to a cage in the case of particle suspensions). Second, we express mathematically the influence of the confinement on the particle motion by introducing one extra dimension (backbone of the chain in the case of polymers and a radial coordinate inside the cage in the case of particle suspensions) in which diffusive (i.e. dissipative) motion takes place. In this paper we make this two steps on the mesoscopic level on which Maffettone and Minale formulated [10] their original rheological model of suspensions of 
deformable particles. The resulting extended model retains the relative simplicity of the original Maffettone-Minale model (MM-model) but its predictions move closer toward the observed rheological and morphological behavior of concentrated colloids (see e.g. [11]). The extended MM-model is in fact a framework that can be filled with many different physical insights. Rheological and morphological predictions of the extended MM-model are illustrated on one example in which the internal energy is a sum of two terms, one is proportional to the surface area of the particle, and the other expresses the particle-particle interactions. We hope to formulate a multiscale model (i.e. a family of mutually related MM-models with varying amount of details of the physics involved) in future papers. With such multiscale model we then expect to be able to identify details of the physics playing an essential role in determining rheological and morphological behavior of colloids and transform the qualitative agreement with experimental results that we see in this paper with the MM- and EMMmodels into a quantitative agreement. Finally, we emphasize that the mesoscopic modeling explored in this paper is complementary to direct simulations (e.g. [16]) in which the point of departure is the physics of individual particles.

\section{Acknowledgment}

One of the authors (M.G.) acknowledges the financial support provided by the Natural Sciences and Engineering Research Council of Canada.

\section{References}

[1] J. Mewis, N.J. Wagner, Colloidal Suspension Rheology, Cambridge U.P. Cambridge, 2012.

[2] S.R. Derkash, Rheology of emulsions, Adv. Colloid Interface Sci. 151 (2009) $1-23$.

[3] G.L. Hand, A theory of anisotropic fluids, J. Fluid Mech. 13 (1962) 33-46.

[4] M. Doi, T. Ohta, Dynamics and rheology of complex interfaces, J. Chem. Phys. 95 (1991) 1242-1248.

[5] M. Grmela, A. Ait-Kadi, Comments on the Doi-Ohta theory of blends, J. NonNewtonian Fluid Mech. 55 (1994) 191-195.

[6] M. Grmela, A. Ait-Kadi, Rheology of inhomogeneous immiscible blends, J. NonNewtonian Fluid Mech. 77 (1998) 191-199.

[7] F.M. Gu, M. Grmela, Flow properties of immiscible Blemds: Doi-Ohta Model with active advection, Phys. Rev. E 78 (2005) 056302.

[8] B.J. Edwards, M. Dressler, A rheological model with constant approximate volume for immiscible blends of ellipsoidal droplets, Rheol. Acta 42 (2003) 326-337.

[9] N.J. Wagner, H.C. Öttinger, B.J. Edwards, Generalized Doi-Ohta Model for multiphase flow developed via GENERIC, AIChE J. 45 (1999) 1169.

[10] P.M. Maffettone, M. Minale, Equations of change for ellipsoidal drops in viscous flow, J. NonNewtonian Fluid Mech. 78 (1998) 227.

[11] S. Khandavalli, J.P. Rothstein, Extensional rheology of shear-thickening fumed silica nanoparticles dispersed in an aqueous polyethylene oxide solution, J. Theol. 58 (2014) 411-431.
[12] K. van der Vaart, Y. Rahmani, R. Zargar, Z. Hu, D. Bonn, P. Shall, Rheology of concentrated soft and hard-sphere suspensions, J. Rheol. 57 (2013) 11951209.

[13] P.G. deGennes, Reptation of a polymer chain in the presence of fixed obstacles, J. Chem. Phys. 55 (1971) 572-579.

[14] S.F. Edwards, Configurations and dynamics of the polymer chain, in: R. Balin, G. Weill, (Eds.), Molecular fluids, Gordon and Breach, London, 1976, pp.151-208.

[15] P.N. Pusey, Colloidal glasses, J. Phys.: Condens Mattet 20 (2008) $494202-$ 494208.

[16] N. Koumakis, J.F. Brady, G. Petekidis, Complex oscillatory yielding of model hard-sphere glasses, Phys. Rev. Lett. 110 (2013) 178301.

[17] A. Clebsch, Über die Integration der hydrodynamische Gleichungen, J. Reine Angew. Math 56 (1859) 1-10.

[18] J.E. Marsden, A. Weinstein, Physica D 7 (1983) 305.

[19] V.L. Ginzburg, L.D. Landau, On the theory of superconductivity, Zhur. Eksp. Theor. Fiz. 20 (1950) 1064-1082.

[20] J.W. Cahn, J.E. Hilliard, J. Chem. Phys. 28 (1958) 258.

[21] H. Ziegler, An Introduction to Thermomechanics: Field Theory and Variational Principles, North-Holland, Amsterdam, New Yourk, Oxford, 1997.

[22] I.E. Dzyaloshinskii, G.E. Volovick, Poisson brackets in condense matter physics, Ann. Phys. (NY) 125 (1980) 67-97.

[23] M. Grmela, Particle and bracket formulations of kinetic equations, Contemporary Math. 28 (1984) 125-132.

[24] A.N. Kaufman, Dissipative Hamiltonian systems: a unifying principle, Phys. Letters A 100 (1984) 419.

[25] P.J. Morrison, Bracket formulation for irreversible classical fields, Phys. Lett. A 100 (1984) 423.

[26] M. Grmela, Bracket formulation of diffusion-convection equations, Physica D 21 (1986) 179-212.

[27] M. Grmela, H.C. Öttinger, Dynamics and thermodynamics of complex fluids: general formulation, Phys.Rev.E 56 (1997) 6620-6633.

[28] Öttinger, M. Grmela, Dynamics and thermodynamics of complex fluids: illustration of the general formalism, Phys. Rev. E 56 (1997) 6633-6650.

[29] M. Grmela, Role of thermodynamics in multiscale physics, Comput. Math. Appl. 65 (2013) 1457-1470.

[30] M. Grmela, Bracket formulation of dissipative time evolution equations, Phys. Lett. A 111 (1985) 36-40.

[31] B.J. Edwards, M. Dressler, M. Grmela, A. Ait-kadi, Rheological models with microstructural constraints, Rheol. Acta 42 (2003) 64-75.

[32] A. Ait-Kadi, A. Ramazani, M. Grmela, C. Zhou, Volume preserving rheological models for polymer melts and solutions using GENERIC formalism, J. Rheol. 43 (1999) 51-73.

[33] R.J. Gordon, W.R. Showalter, Anisotropic fluid theory: a different approach to the dumbell theory of dilute polymer solutions, Trans. Soc. Rheol. 16 (1972) 79-97.

[34] M. Grmela, Why GENERIC?, J NonNewtonian Fluid Mech. 165 (2010) 980-986.

[35] J.F. Gu, M. Grmela, GENERIC model of active advection, J. NonNewtonian Fluid Mech. 152 (2008) 12-26.

[36] M. Grmela, A. Ammar, F. Chinesta, Extra stress tensor in fiber suspensions: mechanics and thermodynamics, J. Rheol. 55 (2011) 17-24.

[37] L.P. Bouchaud, J. Phys. 12 (1992) 1705.

[38] C. Monthus, J.P. Bouchaud, J. Phys. A 29 (1996) 3847.

[39] P. Sollich, Rheological constitutive equation for a model of soft glassy materials' Phys, Rev. E. 58 (1997) 738-757.

[40] R.B. Bird, O. Hassager, R.C. Armstrong, C.F. Curtiss, Dynamics of Polymeric Fluids, vol. 1-2, John Wiley and Sons, 1977.

[41] H. Eslami, M. Grmela, Mesoscopic formulation of reptation Rheol, Acta 47 (2008) 399-415.

[42] J.F. Gu, M. Grmela, M. Bousmina, A mesoscopic rheological model of immiscible blends with the interface covered with a surface active agent, Phys. Fluids 20 (2008) 043102. 